\title{
Increased procalcitonin level is a risk factor for prolonged fever in children with Mycoplasma pneumonia
}

Ji Eun Jeong, MD, Ji Eun Soh, MD, Ji Hee Kwak, MD, Hye Lim Jung, MD, PhD, Jae Won Shim, MD, PhD, Deok Soo Kim, MD, PhD, Moon Soo Park, MD, PhD, Jung Yeon Shim, MD, PhD

Department of Pediatrics, Kangbuk Samsung Hospital, Sungkyunkwan University School of Medicine, Seoul, Korea

Purpose: Macrolide-resistant Mycoplasma pneumoniae pneumonia (MPP) is characterized by prolonged fever and radiological progression despite macrolide treatment. Few studies have examined serum procalcitonin (PCT) level in children with MPP. We aimed to investigate the association of acute inflammation markers including PCT with clinical parameters in children with MPP.

Methods: A total of 147 children were recruited. The diagnosis of MPP relied on serial measurement of IgM antibody against mycoplasma and/or polymerase chain reaction. We evaluated the relationships between C-reactive protein (CRP), PCT, and lactate dehydrogenase (LDH) levels and white blood cell (WBC) counts, and clinical severity of the disease. We used multivariate logistic regression analysis to estimate the odds ratio for prolonged fever ( $>3$ days after admission) and hospital stay ( $>6$ days), comparing quintiles $2-5$ of the PCT levels with the lowest quintile.

Results: The serum PCT and CRP levels were higher in children with fever and hospital stay than in those with fever lasting $\leq 3$ days after admission and hospital stay $\leq 6$ days. CRP level was higher in segmental/ lobar pneumonia than in bronchopneumonia. The LDH level and WBC counts were higher in children with fever lasting for $>3$ days before compared to those with fever lasting for $\leq 3$ days. The highest quintile of PCT levels was associated with a significantly higher risk of prolonged fever and/or hospital stay than the lowest quintile.

Conclusion: Serum PCT and CRP levels on admission day were associated with persistent fever and longer hospitalization in children with MPP.

Key words: Mycoplasma pneumoniae, Pneumonia, Procalcitonin, Drug resistance, Child

\section{Introduction}

Mycoplasma pneumoniae pneumonia (MPP) is one of the most common communityacquired pneumonia (CAP) in children and adolescents. ${ }^{1)}$ Even though MPP is usually mild and self-limiting, it can progress into a life-threatening, fulminant pneumonia. ${ }^{2-4)}$ Outbreaks of MPP occur in 3- to 4-year intervals worldwide, ${ }^{5)}$ and the most recent epidemics in Korea happened in 2006-2007, 2011, and 2015-2016.6)

Macrolides have been used as a first-line antibiotic treatment in children with MPP. However, since 2003, macrolide-resistant MPP (MRMP) has become more common, especially in East Asian countries such as Korea, China, and Japan. ${ }^{7,8)}$ The prevalence of MRMP in Korea reached up to $84.6 \%$ in 2015. ${ }^{9-11)}$ Fever is considered an important indicator of antibiotic responsiveness in CAP. It has been reported that fever lasts longer in patients with MRMP than in patients with macrolide-susceptible MPP during treatment with macrolides. Some
Corresponding author: Jung Yeon Shim, MD, PhD Department of Pediatrics, Kangbuk Samsung Hospital, Sungkyunkwan University School of Medicine, 29 Saemunan-ro, Jongno-gu, Seoul 03181, Korea Tel: $+82-2-2001-2200$

Fax: +82-2-2001-2199

E-mail: jy7.shim@samsung.com https://orcid.org/0000-0001-9367-2233

Received: 13 September, 2017

Revised: 10 November, 2017

Accepted: 21 November, 2017

Copyright ( 2018 by The Korean Pediatric Society

This is an open-access article distributed under the terms of the Creative Commons Attribution NonCommercial License (http://creativecommons.org/ licenses/by-nc/4.0// which permits unrestricted noncommercial use, distribution, and reproduction in any medium, provided the original work is properly cited. 
studies reported higher laboratory values, in particular for lactate dehydrogenase (LDH) and C-reactive protein (CRP), in patients with MRMP. ${ }^{12,13)}$ Even though several clinical studies aimed to identify an indicator for MRMP, no consistent results have been obtained.

Procalcitonin (PCT), a precursor of thyroid hormone, has been used as a biomarker of bacterial infection. Serum PCT level increases within 2-4 hours and peaks within 6-24 hours of systemic infection, earlier than CRP level. ${ }^{14)}$ PCT concentration parallels the severity of infection. ${ }^{15)}$ This may help distinguish a severe bacterial infection from a milder bacterial or viral infection. Many studies have investigated the relationship of serum PCT level with pneumonia, which may be instrumental in establishing diagnosis, determining prognosis, and guiding the decision to administer an empirical antibiotic therapy or to assess the effectiveness of antibiotics. ${ }^{16,17)}$ However, only a few studies have been conducted on PCT levels in children with MPP. ${ }^{18)}$ We measured the levels of acute inflammatory markers, such as PCT, LDH, and CRP, and white blood cell (WBC) counts in patients with MPP and investigated the relationships between these parameters and attributes of clinical severity, including pneumonia type, fever duration, and hospitalization.

\section{Materials and methods}

\section{Study subjects}

This prospective cross-sectional study was performed from August 2015 to March 2016 in the Pediatric Department, Kangbuk Samsung Hospital, Seoul, Korea. We enrolled 147 children who were admitted to the hospital because of CAP and diagnosed with MPP. The diagnosis of CAP was based on clinical symptoms and signs of lower respiratory infection, as well as on chest radiography findings. MPP was defined as follows: (1) positive serum IgM antibody against mycoplasma, with an increase in optical density (OD) or seroconversion of IgM antibody from negative to positive values after 4-7 days, and/or (2) positive results of polymerase chain reaction (PCR) for mycoplasma in samples from nasopharyngeal aspiration or sputum samples. Children who had a history of tuberculosis, chronic pulmonary diseases, congenital heart diseases, immunodeficiency, immunosuppressive conditions or medications, or congenital anomalies were excluded from the study.

All the children were treated with oral azithromycin from the first day of admission. We examined the patients' age, sex, hospital stay, durations of fever before and after admission, chest radiographs, serum PCT, CRP, and LDH levels, and WBC counts. Prolonged fever was defined as persistent fever for more than 3 days after admission, and prolonged hospital stay was defined as hospitalization for more than 6 days. Pneumonia was classified as bronchopneumonia and segmental/lobar pneumonia by 2 radiologists.

The study protocol was approved by the Institutional Review Board of the Kangbuk Samsung Hospital (KBSMC201509017003-
HE003), and informed consent was obtained from the children's guardians.

\section{Laboratory tests}

Blood tests for measuring peripheral WBC counts, and CRP, LDH, and mycoplasma IgM antibody levels were performed on admission. A blood test for measuring mycoplasma IgM antibody level was performed within 7 days after admission for diagnosing MPP.

A serum sample for PCT level determination was collected on admission and stored at $-20^{\circ} \mathrm{C}$ until analyzed. Serum PCT level was measured on a Roche Cobas e601 analyzer based on an electrochemiluminescence immunoassay using the Elecsys BRAHMS PCT kit (Roche Diagnostics Korea, Seoul, Korea). The assay detection range was 0.02-100 ng/mL.

Serum mycoplasma IgM level was assayed using an enzyme immunoassay (RIDASCREEN; R-Biopharm AG, Darmstadt, Germany). The cutoff level for mycoplasma IgM antibody level was 71 $\mathrm{U} / \mathrm{mL}$. Mycoplasma PCR of sputum or nasopharyngeal aspiration samples was performed using the Bio-Core M. pneumoniae PCR Kit (Seegene, Seoul, Korea). The mycoplasma PCR was performed in 2 patients, and those patients showed positive results in both PCR and serology tests. PCR was not performed in the remaining patients, and the diagnosis was established using the enzyme immunoassay (EIA) method.

\section{Statistical analyses}

Statistical analyses were performed using Stata ver. 14.2 (Stata Corp LLC, College Station, TX, USA). Mean values were compared between 2 groups using the Student $t$ test. Significance of differences between groups in categorical variables was tested using chi-square analysis. For nonnormally distributed data, a Mann-Whitney test was performed. We used a multivariate logistic regression analysis to estimate odds ratio (OR) for prolonged fever duration and hospital stay; quintiles 2-5 of serum PCT, CRP, and LDH levels were compared to the lowest quintiles after adjusting for age and sex. All data are expressed as mean \pm standard deviation or median and interquartile range (IQR), and significance was defined as a $P$ value of $<0.05$.

\section{Results}

\section{Patient characteristics}

The baseline characteristics of the study subjects are presented in Table 1. We included 147 children in this study. The mean age was 5.9 years (IQR, 3-8 years), and boys comprised 52.4\% of the subjects. Serum of all the 147 patients was positive for IgM antibody against mycoplasma. PCR analysis detected mycoplasma in two patients. The mean durations of fever before and after admission were 4.6 and 2.7 days, respectively. The mean hospital stay was 6.7 days (IQR, 5-8 days). The mean LDH level was 312 IU/L, the median 
Table 1. Characteristics of study subjects $(n=147)$

\begin{tabular}{|c|c|}
\hline Characteristic & Value \\
\hline Age (yr) & $5.9(3-8)$ \\
\hline Male sex & $77(52.4)$ \\
\hline White blood cell counts $\left(/ \mathrm{mm}^{3}\right)$ & $8,474 \pm 3,092$ \\
\hline Lymphocyte counts $\left(/ \mathrm{mm}^{3}\right)$ & $2,207 \pm 1,242$ \\
\hline Duration of fever before admission (day) & $4.6(3-6)$ \\
\hline Duration of fever after admission (day) & $2.7(1-4)$ \\
\hline Hospital day (day) & $6.7(5-8)$ \\
\hline Lactate dehydrogenase (IU/L) & $312 \pm 67$ \\
\hline C-reactive protein (mg/dL) & $1.99(0.77-3.88)$ \\
\hline Procalcitonin (ng/mL) & $0.05(0.02-0.09)$ \\
\hline \multicolumn{2}{|l|}{ Type of pneumonia } \\
\hline Broncho & $32(21.7)$ \\
\hline Segmental/lobar & $115(78.2)$ \\
\hline \multicolumn{2}{|l|}{ Effusion } \\
\hline No effusion & $142(96.6)$ \\
\hline Effusion & $5(3.4)$ \\
\hline \multicolumn{2}{|l|}{ Fever duration before admission } \\
\hline 3-5 Days & $42(28.5)$ \\
\hline 6-7 Days & $39(26.5)$ \\
\hline >7 Days & $36(24.5)$ \\
\hline \multicolumn{2}{|l|}{ Fever duration after admission } \\
\hline$\leq 3$ Days & $77(52.4)$ \\
\hline >3 Days & $70(47.6)$ \\
\hline
\end{tabular}

Values are presented as mean (interquartile range), number (\%), or mean \pm standard deviation

of CRP level was $1.99 \mathrm{mg} / \mathrm{dL}$, and the PCT level was $0.05 \mathrm{ng} / \mathrm{mL}$. The prevalence rates of segmental/lobar pneumonia and parapneumonic effusion were 78.8\% and 3.4\%, respectively. The percentage of children who had fever for $>7$ days before admission was $24.5 \%$. In total, $47.6 \%$ of the children had prolonged fever after admission. For patients who had persistent fever and no improvement on chest Xray radiography, the antibiotic was changed to levofloxacin (6 cases) and corticosteroids were added (110 cases). (Data not shown in the table)

\section{Serum levels of PCT, LDH, and CRP and WBC counts according to fever duration, hospital stay length, and types of pneumonia}

We compared serum PCT, LDH, and CRP levels and WBC counts between patients with different types of pneumonia, durations of fever before and after admission, and lengths of hospital stay. Since serum PCT and CRP levels were not distributed normally, median values and IQRs were used instead of means and standard deviations, and the Mann-Whitney test was employed for statistical analysis. We found no significant differences in serum PCT levels according to type of pneumonia, number of lobes involved, presence of effusion, or duration of fever before admission. However, the mean serum PCT level was higher in children with prolonged fever ( $>3$ days after admission) than in those with fever lasting for $\leq 3$ days after admission $(P=0.01)$. In addition, children who were admitted to the hospital for $>6$ days had higher serum PCT levels than those who stayed in the hospital for $\leq 6$ days $(P=0.02)$. Serum CRP levels were significantly increased in children with segmental/lobar pneumonia compared to those with bronchopneumonia. Patients with prolonged fever ( $>3$ days) and longer hospitalization ( $>6$ days) had higher CRP levels ( $P=0.03$ and $P=0.01$, respectively). In contrast, serum LDH levels and WBC counts were higher in children who had fever for $>3$ days before admission $(P=0.01)$. However, no significant differences were found in serum LDH levels and WBC counts in children with different fever durations after admission or lengths of hospital stay (Table 2).

\section{Logistic regression analysis of serum PCT, LDH, and CRP levels for prolonged fever and hospital stay length}

We assessed serum PCT, LDH, and CRP levels for prolonged fever (Table 3) and hospital stay length (Table 4) by using a multivariate logistic regression analysis. The highest quintile of PCT and CRP levels was associated with prolonged fever (OR, 3.69; 95\% confidence interval [CI], 1.27-10.7 and OR, 4.03; 95\% CI, 1.21-13.34, respectively) and hospital stay length (OR, 3.85; 95\% CI, 1.16-12.79 and OR, 10.78; 95\% CI, 7.62-18.18, respectively) as compared with the lowest quintile. However, we found no association between serum LDH level on admission and prolonged fever or hospital stay length.

\section{Discussion}

In the present study, we found that patients with prolonged fever after admission (defined as persistent fever for $>3$ days after initiation of macrolide therapy) and longer hospitalization (defined as hospital stay of $>6$ days) had higher serum PCT and CRP levels on admission. Although the increasing incidence of MRMP is believed to be a major cause of severe morbidity, refractory pneumonia is caused not only by MRMP but also excessive host immune reaction against the insults from the MP infection. ${ }^{19)}$ It has been suggested that not only pathogens but also excessive host immune reaction are responsible for acute lung injury. Thus, early administration of immunomodulators such as corticosteroids or intravenous immunoglobulin may induce a rapid improvement of clinical symptoms and radiological signs in refractory MPP ${ }^{20)}$ We treated the majority of patients who had fever for $>3$ days after initiation of macrolide therapy with corticosteroids, and small percentage of patients were treated with levofloxacin, as described in the Result section.

Emerging MRMP is associated with mutations at sites 2063, 2064, 2067, and 2617 in domain $V$ of the $23 \mathrm{~S}$ rRNA sequence. ${ }^{9,21)}$ Some patients with MRMP present with persistent fever, have a prolonged 
Table 2. Comparison of serum PCT, LDH levels, CRP, and WBC according to types of pneumonia, fever duration before and after admission, and hospital stay length

\begin{tabular}{|c|c|c|c|c|c|c|c|c|}
\hline Variable & PCT (ng/mL) & $P$ value & LDH (IU/L) & $P$ value & $\mathrm{CRP}(\mathrm{mg} / \mathrm{dL})$ & $P$ value & WBC $\left(/ \mathrm{mm}^{3}\right)$ & $P$ value \\
\hline \multicolumn{9}{|l|}{ Type of pneumonia } \\
\hline Broncho (n=32) & $0.04(0.02-0.06)$ & 0.22 & $306 \pm 54$ & 0.55 & $0.89(0.60-2.36)$ & 0.01 & $8,251 \pm 3,412$ & 0.64 \\
\hline Segmental/lobar $(n=115)$ & $0.05(0.03-0.1)$ & & $314 \pm 70$ & & $2.54(1.10-4.21)$ & & $8,536 \pm 3,010$ & \\
\hline \multicolumn{9}{|l|}{ Number of lobes } \\
\hline $1(n=102)$ & $0.05(0.03-0.10)$ & 0.34 & $313 \pm 69$ & 0.49 & $2.48(1.04-4.18)$ & 0.19 & $8,454 \pm 3,045$ & 0.40 \\
\hline$\geq 2(n=13)$ & $0.08(0.04-0.10)$ & & $327 \pm 79$ & & $3.26(1.66-5.31)$ & & $9,197 \pm 2,596$ & \\
\hline \multicolumn{9}{|l|}{ Effusion } \\
\hline No effusion ( $n=142$ ) & $0.05(0.03-0.09)$ & 0.64 & $307 \pm 58$ & 0.00 & $1.98(0.76-3.81)$ & 0.43 & $8,474 \pm 3,127$ & 0.99 \\
\hline Effusion $(n=5)$ & $0.07(0.04-0.10)$ & & $460 \pm 126$ & & $3.9(1.78-4.04)$ & & $8,488 \pm 2,095$ & \\
\hline \multicolumn{9}{|c|}{ Duration of fever before admission (day) } \\
\hline$\leq 3(n=30)$ & $0.04(0.02-0.08)$ & 0.29 & $283 \pm 43$ & 0.01 & $2.19(0.76-4.31)$ & 0.82 & $8,096 \pm 2,776$ & 0.01 \\
\hline$>3(n=117)$ & $0.05(0.03-0.09)$ & & $319 \pm 70$ & & $1.88(0.85-3.72)$ & & $9,948 \pm 3,806$ & \\
\hline \multicolumn{9}{|c|}{ Duration of fever after admission (day) } \\
\hline$\leq 3(n=77)$ & $0.04(0.02-0.07)$ & 0.01 & $317 \pm 61$ & 0.39 & $1.4(0.6-3.57)$ & 0.03 & $8,694 \pm 3,388$ & 0.36 \\
\hline$>3(n=70)$ & $0.06(0.04-0.11)$ & & $307 \pm 73$ & & $2.60(1.13-4.31)$ & & $8,232 \pm 2,734$ & \\
\hline \multicolumn{9}{|l|}{ Hospital stay (day) } \\
\hline$\leq 6(n=55)$ & $0.04(0.02-0.07)$ & 0.02 & $312 \pm 50$ & 0.94 & $1.15(0.6-2.64)$ & 0.01 & $8,434 \pm 3,588$ & 0.90 \\
\hline$>6(n=92)$ & $0.06(0.03-0.11)$ & & $312 \pm 76$ & & $2.93(1.17-4.68)$ & & $8,498 \pm 2,775$ & \\
\hline
\end{tabular}

Values are presented as median (interquartile range) or mean \pm standard deviation.

PCT, procalcitonin; LDH, lactate dehydrogenase; CRP, C-reactive protein; WBC, white blood cell.

Table 3. Logistic regression of serum procalcitonin and lactate dehydrogenase for prolonged fever more than 3 days after admission

\begin{tabular}{|c|c|c|c|c|c|}
\hline Variable & 1st quintile & 2nd quintile & 3rd quintile & 4th quintile & 5th quintile \\
\hline \multicolumn{6}{|l|}{ PCT (ng/mL) } \\
\hline Median & 0.02 & 0.034 & 0.055 & 0.077 & 0.168 \\
\hline OR (95\% Cl) & & $0.94(0.33-2.87)$ & $2.13(0.73-6.16)$ & $1.45(0.51-4.09)$ & $3.69(1.27-10.70)$ \\
\hline \multicolumn{6}{|l|}{ LDH (IU/mL) } \\
\hline Mean & 248 & 275.5 & 298 & 327 & 395 \\
\hline OR (95\% Cl) & & $0.48(0.17-1.36)$ & $0.51(0.17-1.55)$ & $0.52(0.17-1.51)$ & $0.50(0.17-1.41)$ \\
\hline \multicolumn{6}{|l|}{ CRP (mg/dL) } \\
\hline Median & 0.6 & 1.06 & 2.05 & 3.57 & 5.94 \\
\hline OR (95\% Cl) & & $2.12(0.67-6.62)$ & $4.02(1.28-12.62)$ & $2.42(0.79-7.39)$ & $4.03(1.21-13.34)$ \\
\hline
\end{tabular}

Adjusted for age and sex.

PCT, procalcitonin; LDH, lactate dehydrogenase; CRP, C-reactive protein; OR, odds ratio; Cl, confidence interval.

hospital stay, and can have a high risk of complications. ${ }^{22,23)}$ Therefore, biomarkers to predict refractory MPP can be useful for making early decisions in therapeutic planning.

Serum PCT level has been studied as an early diagnostic indicator to predict the progression of bacterial infections or sepsis. ${ }^{24)}$ Among other markers such as CRP level and WBC count, serum PCT level has been considered the most useful marker for differentiation of bacterial pneumonia. ${ }^{25)}$ Among adult patients with CAP, those with high pneumonia severity indices have high PCT levels. A recent study of serum PCT levels in hospitalized children with CAP suggested that low PCT concentrations $(<0.1 \mathrm{ng} / \mathrm{mL})$ were not associated with a typical bacterial pneumonia, which may help reduce the need for antibiotic treatments. ${ }^{26)}$ However, studies of PCT levels in pneumonia mostly investigate associations with typical bacterial pathogens such as Streptococcus pneumoniae and Haemophilus influenzae, ${ }^{27)}$ while PCT levels in MPP, especially in refractory cases, have not been thoroughly studied.

In this study, the median levels of PCT and CRP were $0.05 \mathrm{ng} /$ $\mathrm{mL}$ and $1.99 \mathrm{mg} / \mathrm{dL}$, respectively. This finding suggests that $\mathrm{M}$. pneumoniae infection is more likely to be a viral infection than a bacterial infection. Furthermore, in PCT-guided antibiotic therapy, the use of antibiotics is discouraged in patients with PCT levels $<0.1$ $\mathrm{ng} / \mathrm{mL} .{ }^{15)}$ In this study, the serum PCT concentration was low, but all the samples were within the detectable range. Although low PCT 
Table 4. Logistic regression of serum procalcitonin and lactate dehydrogenase for hospital days more than 6 days

\begin{tabular}{|c|c|c|c|c|c|}
\hline Variable & 1st quintile & 2nd quintile & 3rd quintile & 4th quintile & 5th quintile \\
\hline \multicolumn{6}{|l|}{ РCT (ng/mL) } \\
\hline Median & 0.02 & 0.034 & 0.055 & 0.077 & 0.168 \\
\hline OR (95\% Cl) & & $0.83(0.30-2.34)$ & $1.28(0.44-3.71)$ & $0.92(0.33-2.54)$ & $3.85(1.16-12.79)$ \\
\hline \multicolumn{6}{|l|}{ LDH (IU/mL) } \\
\hline Mean & 248 & 275.5 & 298 & 327 & 395 \\
\hline OR (95\% Cl) & & $0.63(0.21-1.85)$ & $0.91(0.28-2.86)$ & $0.57(0.19-1.75)$ & $0.68(0.23-2.02))$ \\
\hline \multicolumn{6}{|l|}{ CRP (mg/dL) } \\
\hline Median & 0.60 & 1.06 & 2.05 & 3.57 & 5.94 \\
\hline OR (95\% Cl) & & $10.3(2.96-35.9)$ & $6.00(1.81-19.96)$ & 8.19 (2.48-27.02) & 10.78 (7.62-18.18) \\
\hline
\end{tabular}

Adjusted for age and sex.

PCT, procalcitonin; LDH, lactate dehydrogenase; CRP, C-reactive protein; $\mathrm{OR}$, odds ratio; $\mathrm{Cl}$, confidence interval.

levels can distinguish atypical pneumonia from typical bacterial pneumonia, MPP treatment requires the use of antibiotics.

MPP is mild and sometimes self-limiting disease, although fatal and life-threatening cases were reported. ${ }^{28)}$ Clinical and laboratory characteristics of fulminant mycoplasma pneumonia include severe cough, high fever, dyspnea, diffuse abnormal findings on chest Xray radiography, and elevated serum CRP levels and WBC counts. ${ }^{29)}$ In this series, there were no patients with severe progressive pneumonia, but we found that the levels of PCT and CRP were higher in patients with pneumonia characterized by prolonged fever and a severe pneumonia pattern. Even though serum PCT and CRP levels were lower than the values typical of a bacterial infection, they may be useful for predicting severe cases of MPP.

A key determining factor of refractory MPP is persistent fever and radiological progression after antibiotic therapy ${ }^{22)}$ In our study, serum PCT level on admission was associated with persistent fever and longer hospital stay. Patients with antibiotic-resistant infections require significantly longer hospital stay; data show that the duration of hospital stay for patients with antibiotic-resistant infections was prolonged by 6.4 to 12.7 days. ${ }^{29)}$ Studies on refractory MPP have shown an increase in serum levels of LDH, CRP, IL-6, or IL18. ${ }^{12,13,30)}$ A recent study showed that excessive serum levels of $\mathrm{LDH}$, CRP, and IL-6 might be early predictors of refractory pneumonia in children with MPP. ${ }^{31}$ We also found that the serum LDH level was elevated in children with pleural effusion or those with persistent fever for $>3$ days before admission, suggesting that severity of lung injury was associated with LDH level. However, serum LDH level was not significantly elevated in the children with prolonged fever after admission or hospital stay. According to our study, serum LDH level on admission is probably not a relevant indicator of refractory pneumonia, in contrast to serum PCT level. There were few patients with refractory pneumonia in this series because of good response to second-line treatments.

The main strength of our study was the assessment of serum PCT levels in children with MPP and the analysis of the association with clinical responsiveness to macrolides. To our knowledge, no study has assessed serum PCT levels in MPP based on fever or hospitalization duration. Though low, the serum PCT concentrations in all the samples were within the detectable range. Low PCT levels can also distinguish atypical pneumonia from typical bacterial pneumonia and help choose suitable antibiotic treatments in children hospitalized for CAP.

Our study has limitations. First, we did not identify the point mutations characteristic of MRMP. Thus, the results cannot be generalized to all patients with MRMP. However, we analyzed serum PCT levels based on clinical responsiveness to antibiotics. Second, disease severity was not assessed, and, while measurement of serum PCT level may have been useful for detecting patients with severe MPP, it may have been less valuable in those with mild MPP. Third, the serum PCT levels were low, mainly because of the association of low PCT levels with atypical bacterial or viral pneumonia. However, the low PCT levels shown in this study could be useful for choosing adequate antibiotics or second-line treatments for children with antibiotic-unresponsive CAP.

In conclusion, laboratory parameters associated with inflammation, including PCT, CRP, and LDH levels and WBC counts, were elevated in MPP, with individual variations. Serum PCT level was not markedly increased, but repeated measurements of serum PCT level may be incorporated into treatment guidelines for children with refractory pneumonia since PCT level might reflect the severity of the disease.

\section{Conflict of interest}

This work used reagents from Roche Diagnostics for the measurement of PCT levels. The funding source had no role in the design, collection, analysis, interpretation of data, or writing of this manuscript. 


\section{References}

1. Waites KB, Talkington DF. Mycoplasma pneumoniae and its role as a human pathogen. Clin Microbiol Rev 2004;17:697-728.

2. Sztrymf B, Jacobs F, Fichet J, Hamzaoui O, Prat D, Avenel A, et al. Mycoplasma-related pneumonia: a rare cause of acute respiratory distress syndrome (ARDS) and of potential antibiotic resistance. Rev Mal Respir 2013;30:77-80.

3. Wang RS, Wang SY, Hsieh KS, Chiou YH, Huang IF, Cheng MF, et al. Necrotizing pneumonitis caused by Mycoplasma pneumoniae in pediatric patients: report of five cases and review of literature. Pediatr Infect Dis J 2004;23:564-7.

4. Shen Y, Zhang J, Hu Y, Shen K. Combination therapy with immunemodulators and moxifloxacin on fulminant macrolide-resistant Mycoplasma pneumoniae infection: a case report. Pediatr Pulmonol 2013;48:519-22.

5. Foy HM, Kenny GE, Cooney MK, Allan ID. Long-term epidemiology of infections with Mycoplasma pneumoniae. J Infect Dis 1979;139: 681-7.

6. Kim EK, Youn YS, Rhim JW, Shin MS, Kang JH, Lee KY. Epidemiological comparison of three Mycoplasma pneumoniae pneumonia epidemics in a single hospital over 10 years. Korean J Pediatr 2015; 58:172-7.

7. Yamazaki T, Kenri T. Epidemiology of Mycoplasma pneumoniae Infections in Japan and therapeutic strategies for macrolide-resistant M. pneumoniae. Front Microbiol 2016;7:693.

8. Hong KB, Choi EH, Lee HJ, Lee SY, Cho EY, Choi JH, et al. Macrolide resistance of Mycoplasma pneumoniae, South Korea, 2000-2011. Emerg Infect Dis 2013;19:1281-4.

9. Morozumi M, Takahashi T, Ubukata K. Macrolide-resistant Mycoplasma pneumoniae: characteristics of isolates and clinical aspects of community-acquired pneumonia. J Infect Chemother 2010;16:78-86.

10. Zhao F, Lv M, Tao X, Huang H, Zhang B, Zhang Z, et al. Antibiotic sensitivity of 40 Mycoplasma pneumoniae isolates and molecular analysis of macrolide-resistant isolates from Beijing, China. Antimicrob Agents Chemother 2012;56:1108-9.

11. Kim JH, Kim JY, Yoo CH, Seo WH, Yoo Y, Song DJ, et al. macrolide resistance and its impacts on M. pneumoniae pneumonia in children: comparison of two recent epidemics in Korea. Allergy Asthma Immunol Res 2017;9:340-6.

12. Inamura N, Miyashita N, Hasegawa S, Kato A, Fukuda Y, Saitoh A, et al. Management of refractory Mycoplasma pneumoniae pneumonia: utility of measuring serum lactate dehydrogenase level. J Infect Chemother 2014;20:270-3.

13. Seo YH, Kim JS, Seo SC, Seo WH, Yoo Y, Song DJ, et al. Predictive value of C-reactive protein in response to macrolides in children with macrolide-resistant Mycoplasma pneumoniae pneumonia. Korean J Pediatr 2014;57:186-92.

14. Becker KL, Snider R, Nylen ES. Procalcitonin assay in systemic inflammation, infection, and sepsis: clinical utility and limitations. Crit Care Med 2008;36:941-52.
15. Christ-Crain M, Müller B. Biomarkers in respiratory tract infections: diagnostic guides to antibiotic prescription, prognostic markers and mediators. Eur Respir J 2007;30:556-73.

16. Korppi M, Remes S, Heiskanen-Kosma T. Serum procalcitonin concentrations in bacterial pneumonia in children: a negative result in primary healthcare settings. Pediatr Pulmonol 2003;35:56-61.

17. Shah SS, Florin TA, Ambroggio L. Procalcitonin in childhood pneumonia. J Pediatric Infect Dis Soc 2018;7:54-5.

18. Moulin F, Raymond J, Lorrot M, Marc E, Coste J, Iniguez JL, et al. Procalcitonin in children admitted to hospital with community acquired pneumonia. Arch Dis Child 2001;84:332-6.

19. Youn YS, Lee KY. Mycoplasma pneumoniae pneumonia in children. Korean J Pediatr 2012;55:42-7.

20. Lee KY. Pneumonia, acute respiratory distress syndrome, and early immune-modulator therapy. Int J Mol Sci 2017;18.

21. Yang HJ, Song DJ, Shim JY. Mechanism of resistance acquisition and treatment of macrolide-resistant Mycoplasma pneumoniae pneumonia in children. Korean J Pediatr 2017;60:167-74.

22. Zhou Y, Zhang Y, Sheng Y, Zhang L, Shen Z, Chen Z. More complications occur in macrolide-resistant than in macrolide-sensitive Mycoplasma pneumoniae pneumonia. Antimicrob Agents Chemother 2014;58:1034-8.

23. Cheong KN, Chiu SS, Chan BW, To KK, Chan EL, Ho PL. Severe macrolide-resistant Mycoplasma pneumoniae pneumonia associated with macrolide failure. J Microbiol Immunol Infect 2016;49:127-30.

24. Maniaci V, Dauber A, Weiss S, Nylen E, Becker KL, Bachur R. Procalcitonin in young febrile infants for the detection of serious bacterial infections. Pediatrics 2008;122:701-10.

25. Hoshina T, Nanishi E, Kanno S, Nishio H, Kusuhara K, Hara T. The utility of biomarkers in differentiating bacterial from non-bacterial lower respiratory tract infection in hospitalized children: difference of the diagnostic performance between acute pneumonia and bronchitis. J Infect Chemother 2014;20:616-20.

26. Stockmann C, Ampofo K, Killpack J, Williams DJ, Edwards KM, Grijalva CG, et al. Procalcitonin accurately identifies hospitalized children with low risk of bacterial community-acquired pneumonia. J Pediatric Infect Dis Soc 2018;7:46-53.

27. Korppi M, Don M, Valent F, Canciani M. The value of clinical features in differentiating between viral, pneumococcal and atypical bacterial pneumonia in children. Acta Paediatr 2008;97:943-7.

28. Izumikawa K. Clinical features of severe or fatal Mycoplasma pneumoniae pneumonia. Front Microbiol 2016;7:800.

29. Ventola CL. The antibiotic resistance crisis: part 1: causes and threats. P T 2015;40:277-83.

30. Narita M, Tanaka H. Cytokines involved in the severe manifestations of pulmonary diseases caused by Mycoplasma pneumoniae. Pediatr Pulmonol 2007;42:397.

31. Zhang Y, Zhou Y, Li S, Yang D, Wu X, Chen Z. The clinical characteristics and predictors of refractory Mycoplasma pneumoniae pneumonia in children. PLoS One 2016;11:e0156465. 